\title{
A comprehensive comparative phylogenomics and demographic evolutionary history of the SARS-CoV-2
}

Özgül Doğan ${ }^{\mathrm{a}}$, Ertan Mahir Korkmaz ${ }^{\mathrm{a}^{*}}$, Mahir Budakª, Battal Çıplak ${ }^{\mathrm{b}}$, Hasan Hüseyin Başıbüyük ${ }^{c}$

a Department of Molecular Biology and Genetics, Faculty of Science, Sivas Cumhuriyet University, Sivas, Turkey.

${ }^{\mathrm{b}}$ Department of Biology, Faculty of Science, Akdeniz University, Antalya, Turkey

'Department of Gerontology, Faculty of Health Sciences, Akdeniz University, Antalya, Turkey.

${ }^{*}$ Corresponding author

E-mail: ekorkmaz@cumhuriyet.edu.tr

\section{ABSTRACT}

A new form of beta coronavirus called severe acute respiratory disease coronavirus type 2 (SARS-CoV-2) causing a recent pandemic outbreak possesses a linear positive ss-RNA genome with a length of 29,903 nt. Here, the genomes of SARS-CoV-2 from 821 samples were characterised for its better understanding of the genomic and evolutionary patterns. The phylogeny of SARS-CoV-2 was reconstructed using concatenated dataset consisting of all peptide encoding sequences under Bayesian Inference (BI) and Maximum Likelihood (ML) approaches. Comparison of all peptide encoding sequences reveals high divergence of amino acid sequences proportional to divergence of nucleotides, indicating that the viral genomic evolution has not been strictly neutral. The most part of the genome was under neutral evolution, however, the specific sites for peptide encoding sequences were evolved under positive selection. As well as providing reliable evidence on transmission routes of the SARSCoV-2 outbreak, the phylogenetics and network analyses suggest the sample reported from Guangdong province is likely ancestor of the pandemic virus form. The overall substitution rate of SARS-CoV-2 genome was estimated to be $1.65 \times 10^{-3}$ per site per year, falling within the range for previously reported RNA viruses. Median estimation of tMRCA from Bayesian coalescent analyses corresponds to 10 September 2019. The exponential growth rate $(r)$, doubling time ( $T d$ ) and $R_{0}$ were estimated to be 47.43 per year, 5.39 days and 2.72, respectively. These findings convincingly emphasise that the use of more comprehensive genome data improves robustness and also enhances understanding of the demographic history of the outbreak.

Keywords: coronavirus, origin, substitution rate, positive selection, demographic dynamics 


\section{INTRODUCTION}

The world is suffering from an ecosystem crisis due to extraordinary population growth and consequent activities of human species. Human populations have experienced a period of explosive growth depending on decreases in external cause mortality during approximately last 250 years following the Industrial Revolution (1). The population of any other species does not increase so rapidly in nature like humans although it is one of the species with the longest generation time. Within the last century, the world population has increased by one billion in each 16 years on average. This continuous growth is closely related to the emergence of modern medicine, and rapid developments in the scientific discoveries which led to technological developments, a relative decrease in competition for food resources and the increased volume of trade as the main results of globalization. The growing human population has also led to anthropogenic impact on the environment promoting continuous and multiple contacts between people, domestic and wild animals. Such contacts were considered as main cause for the emergence and spread of zoonotic infectious diseases in association with crowded settlement (2). Most known examples of the emerging infectious diseases throughout and in various parts of the world include the smallpox, Spanish flu (H1N1 influenza), plague, cholera, Human Immunodeficiency Virus (HIV), hepatitis C, avian flu (H5N1 influenza), swine flu (H1N1 influenza), Severe Acute Respiratory Syndrome Coronavirus (SARS), and Ebola Virus (EBOV) (3-5).

It is known that the emergence and re-emergence of most of the zoonotic infectious diseases are resulted from viruses with RNA as their genetic material, which can rapidly adapt to varying environmental conditions owing to their exceptionally shorter generation times, high mutation rates, frequent recombination and re-assortment events creating novel genotypes from cocirculating strains (quasispecies) $(3,6)$. The occurrence of the high mutation rates, as high as a million times $\left[10^{-2}-10^{-5}\right.$ per site per year (7)] greater than their hosts $\left[2,2 \times 10^{-9}\right.$ per site per year (8)], is considered as a beneficial trait for RNA viruses due to prominent enhancement in the ability of their virulence and evolvability. Despite these exceptionally high mutation rates, most of the mutations are expected to be swept away from viral populations via purifying and/or negative selection. On the other hand, the deterministic nature of the natural selection tends to increase the fitness of the populations favouring beneficial mutations through positive selection in time. The balance between these opposed selective forces ultimately shapes the evolution of the viral populations.

The recent pandemic outbreak resulted from a novel coronavirus, named as SARS-CoV-2 (also referred to as hCoV-19) (9), causing unusual respiratory condition with various degrees of severity was reported for the first time by the end of 2019 in Wuhan, China $(10,11)$. SARSCoV-2 has a linear positive ss-RNA genome with a length of 29,903 nt, consisting of a leader sequence, ORF1ab encoding replicase polyproteins involved in RNA replication and 
transcription processes and non-structural proteins (nsp), S gene encoding spike glycoprotein, E gene encoding envelope protein, $M$ gene encoding membrane glycoprotein, $\mathrm{N}$ gene encoding nucleocapsid phosphoprotein and six ORFs encoding peptides with unknown exact functions (12).

The SARS-CoV-2 is a new strain which has rapidly distributed worldwide within approximately three months infecting millions of people. Yet, there are many unknown aspects of the virus strain which prevent to take plausible measurements and assessments for upcoming of pandemic. One aspect appears to be least concerned by healthcare professional is the past and ongoing evolution of the virus strain. This study primarily aims to estimate the evolution and evolutionary drivers of the virus using available 821 total genomes of SARS-CoV-2 which expands significantly the early findings based on limited number of samples $(13,14)$. Data matrices generated were subjected to appropriate bioinformatics analyses to accomplish the following specific objectives: (i) to determine the place of origin and likely origin strain based on phylogenetic trees, (ii) to estimate the time of origin and the rate of base/amino acid substitutions per individual protein encoding genes and total genome by time estimation analyses, (iii) to characterize the viral genome for base composition, rates of synonymous and nonsynonymous substitutions in connection with presence/absence of selection forces, (iv) to estimate demographic history of virus population based on possible evolutionary drivers, and (v) to determine possible synapomorphic base position states of bat coronavirus and SARSCoV-2 using a strict phylogenetic approach. Finally, a recent evolutionary history of the virus will be discussed in the light of the results.

\section{MATERIAL and METHODS}

\section{Construction of dataset and basic sequence statistics}

A dataset of 821 samples was constructed by retrieving the complete genomes of SARS-CoV2 representing 38 countries from GISAID (13 March 2020; https://www.gisaid.org/) database. All details of the sampling dataset were presented in Table S1. Annotation of the genomes were performed using ORFFinder implemented in Geneious R9 (15) and by comparison with the reference SARS-CoV-2 genome from NCBI (NC045512). Sequences were aligned using MAFFT v7.450 (16) and manually checked by MEGA X (17). The basic sequence statistics such as nucleotide and amino acid identities, nucleotide diversity and nucleotide compositions were estimated by MEGA X.

\section{Model selection, phylogenetic analyses and network reconstruction}

One sample representing bat strain (bat-RaTG13-CoV; MN996532) was included to the dataset as outgroup (18) for model selection and phylogenetic analyses. The best-fit evolution model of the dataset was selected using ModelTest-NG (19), applying the default parameters 
under three statistical criteria (AIC, BIC, and DT). The Maximum Likelihood (ML) tree was built using RAxML v8.0.9 (20) using the GTR substitution model with gamma distribution (gamma shape: 0.507).

To deeply investigate the evolutionary relationships and origin of location of the SARS-CoV-2, a median-joining (MJ) network (21) was constructed using a total of 25 human SARS-CoV-2 samples selected from the basal placement of each clade and/or subclade of the phylogenetic tree generated here including the samples from Wuhan (China), supposed to be origin point of the outbreak (10), and bat-RaTG13-CoV was assigned as outgroup (Table S2). The analysis was performed using the whole genome dataset under the Network v.5.0.1.1 (available at http://www.fluxus-technology.com) with the default settings $(\varepsilon=0)$.

\section{Estimation of mutation rates of genome and genes}

The individually aligned peptide encoding sequences (10 CDS and 16 nsps synthesised from orf1ab) of SARS-CoV-2 samples were concatenated by SequenceMatrix v.1.7.8 (22). The substitution rate estimation of each peptide encoding sequences and whole genome was conducted in BEAST v1.10.4 (23) using the available dates for samples with a random starting tree on the CIPRES science gateway portal (24). As the dataset were not clock-like (likelihood ratio test: $-\operatorname{In}+\mathrm{c}-52308.105,-\operatorname{In}-\mathrm{c}-49863.927$, d.f. $=819, \mathrm{P}=0.0000 \mathrm{E}+000)$, the analyses were performed by an uncorrelated relaxed molecular clock model with a lognormal rate distribution under GTR+G model and combined with a coalescent model with an exponentially growing population [frequently preferred for viral outbreaks and suggested by A. Rambaut (http://virological.org/t/phylodynamic-analysis-176-genomes-6-mar-2020/356)]. Four independent analyses were performed using 100 million generations (sampling every 10000 generations) and then these analyses were combined after removal of the burn-in of $10 \%$ using LogCombiner version 1.10.4. The results were assessed to show convergence and ensure effective sample sizes (ESS > 200) in Tracer v1.7. The maximum clade credibility of trees was calculated using TreeAnnotator v1.10.4 (23) after removing a 20\% burn-in of the samples.

\section{Demographic analyses}

The demographic dynamics of SARS-CoV-2 outbreak were inferred from Bayesian coalescent model using demographic reconstruction option in Tracer v1.7. The doubling time ( $T d)$ was calculated using the following formula: $T d=\ln (2) / r$, assuming that the pandemic is growing exponentially with a constant growth rate $(r)(25)$. The basic reproduction number $\left(R_{0}\right)$ was calculated using the formula by Anderson and May (26): $1+[\ln (2) / T d] \times D$, where $D$ is the duration of the infectious period [13.4 days, (27)]. 
Gene-level selection analyses

To investigate the role of different selective forces in the evolution of each peptide encoding sequences of SARS-CoV-2, the nonsynonymous $(d M)$ and synonymous $(d S)$ substitution rates for each codon, and codons under pervasive and/or episodic positive selection were estimated using the codon-based approaches FUBAR [the Bayesian MCMC-based fast unbiased approximate Bayesian analysis; (28)] and MEME [mixed effects model of evolution; (29)] implemented in HyPhy package (30) on the DataMonkey server (http://www.datamonkey.org/dataupload.php). Significance was assessed by posterior probability $>0.95$ (FUBAR) and $P$-value $<0.05$ (MEME). The magnitude categories (conservative, moderate, and radical) and physicochemical properties of the positively selected sites detected in the result of FUBAR and MEME were identified using the modified MM01 model implemented in TreeSAAP v3.2 (31). Eight categories (1-8) were used to represent the magnitude of substitutions, by setting a sliding window of 20 codons and analysing the properties of 31 amino acids (32). Significant positive z-scores $(P<0.001)$ were accepted as a signal of significant change in function and/or structure.

\section{Comparative distribution of synapomorphies}

The molecular synapomorphic changes that define the clades (or branches) of human SARSCoV-2 and bat-RaTG13-CoV were investigated by generating a new RAxML tree using randomly chosen SARS-CoV-2 genomes of 25 humans and five coronavirus samples [batRaTG13-CoV, two pangolin coronavirus (EPI_ISL_410538, _410721) and two SARS-CoV (AY274119, NC004718)] (see Table S3 for sampling). The sequence data and the ML tree file were imported into PAUP $4.0 \mathrm{~b} 10$ (33). After defining outgroups and selecting maximum parsimony optimality criterion (parsimony settings; character state optimization: DELTRAN), the logfile option was activated (File: 'Log Output to Disk'). Sequence data was then used to obtain a labelled tree reconstruction and a complete list of synapomorphies (Trees: 'Describe Trees' with 'phylogram', 'labelled internal nodes', and 'list of synapomorphies'). The resulting logfile listed all synapomorphies of the dataset. The synapomorphic changes were screened and comparatively analysed in terms of total numbers, positions and substitution types (transitions and transversions) across the genome.

\section{RESULTS and DISCUSSION}

\section{Basic sequence statistics}

It is known that coronaviruses with largest RNA genome exhibit relatively low level of genetic divergence owing to the presence of $3^{\prime}$ exonuclease proofreading activity in their replicases (34). SARS-CoV-2 genomes analysed here displayed a similar pattern. The final length of the 
sequences of human SARS-CoV-2 was 29,238 nt (without outgroups), of which 832 sites were variable. Of these variable sites 232 were parsimony informative while 600 were singleton mutations. Divergence indices for both nucleotide (29,238 nt) and amino acid sequences of concatenated dataset (9746 aa) on average were 0.029 and 0.057 , respectively (Table 1 ). Divergences for each gene were also in low levels ranging from 0.014 (nt div.) and 0.025 (aa div.) in orf7a to 0.070 (nt div.) and 0.120 (aa div.) in E gene. Comparison of all peptide encoding sequences revealed that divergences of amino acid sequences were always higher, proportional to that of nucleotide sequences (Table 1). The greater amino acid divergences were mainly resulted from the high incidence of nucleotide substitutions at non-degenerate and twofold degenerate sites (Table 1), indicating that the viral genome may not be experiencing strictly neutral evolution. If the viral genome has been subjected to the strictly neutral evolution, purifying selection would intensively act on substitutions at non-degenerate sites (35). Additionally, compatible with the recent expansion and relatively short history of the virus, the low nucleotide diversity $(\pi)$ values were observed in each peptide encoding sequences, ranging from 0.00005 in nsp9 to 0.00115 in orf8, with an average of 0.00028 overall mean nucleotide diversity (Table 1 ).

Similar to the previously reported human coronaviruses, SARS-CoV-2 genomes displayed several general patterns in terms of nucleotide composition: preference of (i) $U(32.22 \%)$ over C (18.34\%); (ii) A (29.82\%) over G (19.62\%); (iii) pyrimidines $(50.6 \%)$ over purines $(49.4 \%)$ (Table 2). However, when the codon positions of peptide encoding sequences were analysed separately, a codon bias towards $C$ and $G$ were observed in the first and/or second codon positions of some peptide encoding sequences (Table 2), most probably resulting from the differences between codon preferences.

\section{Phylogeny of SARS-CoV-2}

The phylogeny of SARS-CoV-2 was constructed using the concatenated dataset consisting of all peptide encoding sequences (29,238 nt) by both $M L$ under RAxML and Bayesian Inference (BI) under BEAST. The phylogenetic analyses have recovered trees with almost the same topologies. However, the tree generated under BI approach was more resolved and with higher node supports, therefore, presented and discussed here (Fig. 1). The phylogeny and network analyses provided reliable evidence on transmission routes of the SARS-CoV-2 outbreak. The most basally placed samples were EPI_ISL_413892, _413865,_413855 and _413862 from Guangdong, a southern coastal province of China. The following basally placed samples were (i) the sample from Snohomish County (WA, USA) (EPI_ISL_404895) and this was the first recorded genome/case from US (19 January 2020, Providence Regional Medical Center); (ii) the samples from the provinces of Chongqing (EPI_ISL_408478) and Henan (EPI_ISL_408487) (China). The all remaining virus samples placed into two clearly structured 
main clades with high support [posterior probability $(P P)=1.00$ ] which are denoted clade $A$ and clade B (Fig. 1). The clade A consisted of samples solely from North America. The clade B comprising the rest of samples and subdivided into two well-structured subclades ( $P P=1.00$; Fig. 1), namely "subclade B1" and "subclade B2". The subclade B1 is the most basal one including samples mainly from China, as well as from other Asian countries, Australia and Europe providing information on the first transmission routes of the SARS-CoV-2. The subclade B2 consisting of samples worldwide indicated event of multiple independent transmissions from China. The samples from Europe formed the most recent crown cluster of this subclade. The phylogenetic tree was also largely in congruence with the recently published by Forster et al. (36) and Bai et al. (37), but provided further insights by including higher number of samples, some from previously unsampled countries.

The MJ network revealed that the outgroup bat-RaTG13-CoV sample was connected to the sample from Guangdong (EPI_ISL_413892) with 1132 mutations, which has basal placement also in the phylogenetic tree (Fig. 2). Although Wuhan has widely cited as the origin of outbreak [e.g. $(38,39)$ ], here the sample from Guangdong were consistently recovered as ancestral in both network and phylogenetic analyses. The previous phylogenetic reconstructions either not included the Guangdong's samples (10) or produced outgroup constrained trees assigning Wuhan's samples as reference taxon [e.g. $(36,40)]$. The bat-RaTG13-CoV genome has been regarded as the most recent ancestor of SARS-CoV-2 with the similarity of an average of $96.2 \%$ (10), however, the occurrence of more than 1000 mutations supports the assumption on presumed unknown intermediate host(s) (41). Malayan pangolin was suggested as a potential intermediate host and a recombination event was proposed between pangolin-CoVlike virus and a bat-CoV-RaTG13-like virus for the origin of SARS-CoV-2 (42). The putative ancestral form can be distinguished from the samples of USA (EPI_ISL_404895) and Guangdong (EPI_ISL_413865) with the occurrence of two mutations in S gene: the nonsynonymous mutation $\mathrm{U} 21619 \mathrm{C}$ changing a leucine to a serine and the synonymous mutation U24290C. The other samples with basal placements in the tree (EPI_ISL_413855, _408478, _408487) was also found to be derived from these samples in the network. Similar to the phylogenetic inference, the network indicated an evidence of two evolutionary transmission paths (Fig. 2): (i) from EPI_ISL_413557 (USA) to EPI_ISL_415593 (USA) corresponding to basal samples of the clade $A$ with occurrence of two nonsynonymous mutations in the sequence encoding helicase ( $\mathrm{C} 17654 \mathrm{U}$ replacing a proline to a leucine and A17765G replacing a tyrosine to a cysteine); (ii) from_406801 (Wuhan) to the samples forming the Clade B with occurrence of one synonymous mutation U17967C in the sequence encoding 3'- to- 5' exonuclease.

\section{Estimation of mutation rates of genome and genes}


Estimation of viral mutation rates has crucial importance for tracing evolutionary paths of the virus, and assessing various strategies for developing possible vaccines, antibodies and/or drugs $(43,44)$. Determination of the changes in the mutation rate and mutational spectra can also help deeply understand alterations in viral fitness and virulence, and effects of the host immune system on some virus genotypes (45). The precise mutation rate therefore should be estimated in both genome and gene levels using comprehensive sampling throughout the distribution range of viral infection. The genomic SARS-CoV-2 substitution rate was estimated to be $1.65 \times 10^{-3}$ per site per year $(\mathrm{s} / \mathrm{s} / \mathrm{y})\left(95 \% \mathrm{HPD}=1.44-1.88 \times 10^{-3}\right)($ Table 3$)$ appears to fall within the range previously reported RNA viruses (7). This rate is also moderate when compared to that of other reported coronaviruses (44), but intermedaite to the rates specificic for SARS-CoV-2, lower than that of Giovanetti et al. (13) and Benvenuto et al. (14) $\left(6.58 \times 10^{-}\right.$ ${ }^{3} \mathrm{~s} / \mathrm{s} / \mathrm{y}$ ), higher than reported by A. Rambaut (http://virological.org/t/phylodynamic-analysis176-genomes-6-mar-2020/356) $\left(0.80 \times 10^{-3} \mathrm{~s} / \mathrm{s} / \mathrm{y}\right)$ and fall in to range reported by Bai et al. (37) $\left(95 \% \mathrm{HPD}=1.42 \times 10^{-3}-1.80 \times 10^{-3}\right)$. The estimated substitution rate implied the occurrence of 4 (3.5 to 4.6) new mutations per month in the SARS-CoV-2 genomes. However, the estimated substitution rates of peptide encoding sequences of SARS-CoV-2 exhibited considerable variation, ranging between $9.08 \times 10^{-4} \mathrm{~s} / \mathrm{s} / \mathrm{y}\left(95 \% \mathrm{HPD}=3.22 \times 10^{-5}-2.40 \times 10^{-}\right.$ $\left.{ }^{3}\right)$ in orf $7 \mathrm{~b}$ and $1.15 \times 10^{-2} \mathrm{~s} / \mathrm{s} / \mathrm{y}\left(95 \% \mathrm{HPD}=5.11 \times 10^{-3}-1.88 \times 10^{-2}\right)$ in leader protein (Table 3). The sequences encoding structural proteins displayed higher substitution rates than overall genome, varying from $3.60 \times 10^{-3}$ in $\mathrm{M}$ gene to $8.42 \times 10^{-3}$ in $\mathrm{E}$ gene (Table 3).

\section{Demographic dynamics of the outbreak of SARS-CoV-2}

Median estimation of tMRCA from Bayesian coalescent analyses was found as 2019.69 (95\% HPD = 2019.54 - 2019.83), corresponding to 10 September 2019 (95\% HPD: 19 July 2019 30 October 2019) (Fig. 3). The estimated date was not consistent with the date of first reported case of SARS-CoV-2 (46). The tMRCA date was estimated to be middle of November by A. Rambaut based on only 86 genomes, whereas late September by Bai et al. (37) based on 622 genomes. Independent from any epidemiological information, the exponential growth rate $(r)$ from demographic analyses of the genome data were inferred as 47.43 per year $(95 \%$ HPD $=$ $36.72-66.88$ per year). Based on the growth rate, the $T d$ of the virus was calculated as 5.39 days $(95 \%$ HPD $=3.82-6.96$ days), which is shorter than the rate reported by A. Rambaut (7.2 days). The calculated $T d$ was consistent with the previously reported epidemic doubling times, ranging between 5.0 days (47) and 7.5 days (48). The $R_{0}$ was estimated to be 2.72 (2.33 - 3.43), was mostly in the similar order of magnitude with the reported estimations (11 and references therein), however, higher $R_{0}$ estimations have also been reported $(49,50)$. 
Genomic regions under positive and purifying selection or neutral evolution were visualised estimating the magnitude of $d N$ - $d S$ (Fig. 4). Although the most part of the genome was under purifying selection $(d N<d S)$, the specific sites for peptide encoding sequences were found to be under positive selection $(d N>d S$ ) with significant statistical support (Fig. 4, Table 4). Five sites were detected under episodic positive selection in four peptide encoding sequences by MEME, while only seven sites were found as under pervasive positive selection in four peptide encoding sequences by FUBAR (Table 4). Only two codons in two different peptide encoding sequences (nsp3 and nsp6) were found as positively selected in both approaches, therefore, the TreeSAAP analyses focused only on these two codons. The first one was codon 1179 in the nucleic acid-binding region (NAB) of the replicase protein nsp3, leading to a replacement between alanine and valine. The positive selection at this codon was reported for the first time. This codon was coding threonine in pangolin CoV and serine in BetaCoV England 1. Considering the multiple interactions of NAB with other non-structural proteins such as nsp2, nsp5, orf3a as well as other domains of nsp3 (51), this substitution seems to be significant due to altering both chemical [long-range non-bonded energy $\left(E_{l}\right)$, polar requirement $\left(P_{r}\right)$, polarity $(p)$ and solvent accessible reduction ratio $\left(R_{\alpha}\right)$ ] and structural [beta structure tendency $\left(P_{\beta}\right)$, average number of surrounding residues $\left(N_{s}\right)$ ] properties of the relevant protein (Table 4). The second one was codon 38 in the replicase protein nsp6, locating at the endoplasmic reticulum (ER) and generating autophagosomes (52), with the substitution of leucine to phenylalanine and indicating a signal of structural change altering the property of $P_{\beta}$ of the protein. The substitution in this codon was also reported by Benvenuto et al. (53) and it seems to be variable in other coronaviruses as valine, isoleucine and leucine (52).

\section{Comparison of synapomorphies between bat coronavirus and SARS-CoV-2}

The phylogenetic tree constructed to identify synapomorphies and list of synapomorphic characters were shown in Figure S1 and Table S4, respectively. The ratio of synapomorphic characters to the total number of characters at the branch leading to bat-RaTG13-CoV (2.09\%) was relatively similar to that of the branch leading to SARS-COV-2 (1.88\%). The proportions of transitional substitutions $(78.4 \%$ in bat-RaTG13-CoV and $78.9 \%$ in SARS-COV-2) within these synapomorphic characters were apparently higher than transversions $(21.6 \%$ in batRaTG13-CoV and $21.1 \%$ in SARS-COV-2) at both branches. Although transitions and transversions dispersed similarly throughout the genomes (Fig. 5a), the distribution of transition- and transversion-types exhibited differences (Fig. $5 b$ and $5 \mathrm{c}$ ). Remarkably, A to $\mathrm{G}$ and $\mathrm{U}$ to $\mathrm{C}$ transitions were observed in high frequencies of $63.03 \%$ in bat-RaTG13-CoV and $64.87 \%$ in SARS-CoV-2 on average of total transitions (Fig. 5b). The excessive number of these type of transitions might be related to deamination of adenine to hypoxanthine and 
thermodynamically in favour of hypoxanthine cytosine matches, is also known to be a commonly observed pattern in retroviral and other RNA genomes (54).

\section{CONCLUDING REMARKS}

In the present study, a more comprehensive dataset was utilised than the datasets previously published (86 genomes in A. Rambaut; 622 genomes in Bai et al. (37); and 821 genomes in present study). Although some of the analyses presented here partly correspond to those in previous publications some were conducted for the first time, such as the estimation of substitution rates of each peptide encoding sequences, the inference of demographic evolutionary dynamics ( $R_{0}, T d$ and $r$ ) using genomic data, and definition and comparison of synapomorphies between bat-RaTG13-CoV and SARS-CoV-2. Thus, this study provides more comprehensive information on comparative phylogenomics of SARS-CoV-2 and allow us to reach some basic conclusions.

Firstly, as discussed by Andersen et al. (18), there are two plausible scenarios on the proximal origin of SARS-CoV-2. The first scenario suggests that SARS-CoV-2 has evolved and acquired adaptive traits (mutations in the receptor-binding domain and polybasic cleavage site) in an unknown animal host prior to transmission to human. The second scenario, however, assumed that it has evolved in humans for a period time to gain the adaptive traits before becoming a pandemic form. At present, there is no convincing evidence in favour of either of these, but our results are in line with second scenario providing evidence for the origin of outbreak. Although, Wuhan has frequently been reported as origin place of SARS-CoV-2, our phylogenetic and network analyses strongly suggested that the virus samples from Guangdong province occupies the ancestral position with significant nodal support (Figs. 1 and 2). The Guangdong province is known as the largest import and export centre in China where the Malayan pangolins, a potential intermediate host of SARS-CoV-2, illegally traded (42). This result also challenges the scenarios that the virus transmitted from bats to humans in Wuhan wet market. The second remark is related to origin time of the pandemic form of the SARS-CoV-2. The first cases were announced in December 2019 from China. However, we estimated the date of origin as first half of September 2019, much earlier than the announced date of outbreak. Although, the date of origin estimated here is earlier, but, still close to the date reported by Bai et al. (37). These differences may have resulted from unreported or unidentified asymptomatic cases.

Our third remark is on the evolutionary demographic history of the SARS-CoV-2 during time period of pandemic or the last six months. In general, the whole genome evolved under nearly neutral selection, but the evolution of a few codons departed from neutrality. One codon per each of nsp3 and nsp6 encoding sequences were found to be positively selected in both of FUBAR and MEME analyses. These positively selected codons may enhance the function of 
replicase proteins in favour of shortening the $T d$. The $T d$ estimate of this study (5.39 days; $95 \%$ HPD $=3.82$ - 6.96 days) was similar to some previously reported epidemiologic $T d$ values [5.0 days; (47)], but shorter than others [7.5 days; (48)]. Based on these values, we assume that virus population has evolved towards shorter doubling time and if this particular positive selection pattern continues on nsp3 and nsp6 then further decline in $T d$ may be expected during the upcoming course of the pandemic.

It is also worthwhile to note the decline in the basic reproduction number, the $\mathrm{R}_{0}$, a reference parameter for pathogeny of the agent. Although there has been several reports exceeding approximately $R_{0}>6$, the number estimated here, $\left(R_{0}=2.72\right)$ is amongst the lowest (11 and references therein) and somewhat indicate declining over the time. One of the obvious reason may be the preventive measures taken worldwide. Therefore, it is recommended that measures must be persistent and strongly implemented so that until the declining tendency in $\mathrm{R}_{0}$ becomes perpetual.

Finally, the findings on tMRCA, growth rate, doubling time and $R_{0}$ strongly indicate that investigating more comprehensive genome data improves robustness and also enhances understanding of the demographic history of the outbreak. However, there is still a long way to go in order to better understand the SARS-CoV-2 and its outbreak.

\section{ACKNOWLEDGEMENTS}

This study is dedicated to all healthcare staff and scientists for their diligence and hard work during this SARS-CoV-2 outbreak. We are grateful to the authors and originating and submitting laboratories of the sequences from GISAID's EpiCOV ${ }^{\text {TM }}$ Database. The bioinformatics analyses were partially performed at TUBITAK ULAKBIM, High Performance and Grid Computing Center (TRUBA resources).

\section{REFERENCES}

1. J. Van Bavel, The world population explosion: causes, backgrounds and -projections for the future. Facts, Views Vis. ObGyn 5, 281-291 (2013).

2. S. Cleaveland, D. T. Haydon, L. Taylor, "Overviews of pathogen emergence: Which pathogens emerge, when and why?" in Wildlife and Emerging Zoonotic Diseases: The Biology, Circumstances and Consequences of Cross-Species Transmission, J. E. Childs, J. S. Mackenzie, J. . Richt, Eds. (Current Topics in Microbiology and Immunology, Vol. 315, Springer, 2007), pp. 85-111.

3. R. Carrasco-Hernandez, R. Jácome, Y. López Vidal, S. Ponce de León, Are RNA viruses candidate agents for the next global pandemic? A review. ILAR J. 58, 343-358 (2017).

4. M. E. J. Woolhouse, L. Brierley, C. McCaffery, S. Lycett, Assessing the epidemic 
potential of RNA and DNA viruses. Emerg. Infect. Dis. 22, 2037-2044 (2016).

5. K. E. Jones, et al., Global trends in emerging infectious diseases. Nature 451, 990-993 (2008).

6. P. T. Dolan, Z. J. Whitfield, R. Andino, Mapping the evolutionary potential of RNA viruses. Cell Host Microbe 23, 435-446 (2018).

7. S. Duffy, Why are RNA virus mutation rates so damn high? PLOS Biol. 16, e3000003 (2018).

8. S. Kumar, S. Subramanian, Mutation rates in mammalian genomes. Proc. Natl. Acad. Sci. 99, 803-808 (2002).

9. S. Jiang, et al., A distinct name is needed for the new coronavirus. Lancet 395, 949 (2020).

10. P. Zhou, et al., A pneumonia outbreak associated with a new coronavirus of probable bat origin. Nature 579, 270-273 (2020).

11. D. Wu, T. Wu, Q. Liu, Z. Yang, The SARS-CoV-2 outbreak: What we know. Int. J. Infect. Dis. 94, 44-48 (2020).

12. C. Yin, Genotyping coronavirus SARS-CoV-2: methods and implications. Genomics (2020) https:/doi.org/10.1016/j.ygeno.2020.04.016.

13. M. Giovanetti, D. Benvenuto, S. Angeletti, M. Ciccozzi, The first two cases of 2019nCoV in Italy: Where they come from? J. Med. Virol. 92, 518-521 (2020).

14. D. Benvenuto, et al., The global spread of 2019-nCoV: a molecular evolutionary analysis. Pathog. Glob. Health 114, 64-67 (2020).

15. M. Kearse, et al., Geneious Basic: an integrated and extendable desktop software platform for the organization and analysis of sequence data. Bioinformatics 28, 1647-9 (2012).

16. K. Katoh, D. M. Standley, MAFFT multiple sequence alignment software version 7: improvements in performance and usability. Mol. Biol. Evol. 30, 772-80 (2013).

17. S. Kumar, G. Stecher, M. Li, C. Knyaz, K. Tamura, MEGA X: Molecular evolutionary genetics analysis across computing platforms. Mol. Biol. Evol. 35, 1547-1549 (2018).

18. K. G. Andersen, A. Rambaut, W. I. Lipkin, E. C. Holmes, R. F. Garry, The proximal origin of SARS-CoV-2. Nat. Med. 26, 450-452 (2020).

19. D. Darriba, et al., ModelTest-NG: A new and scalable tool for the selection of DNA and protein evolutionary models. Mol. Biol. Evol. 37, 291-294 (2020).

20. A. Stamatakis, RAxML version 8: A tool for phylogenetic analysis and post-analysis of large phylogenies. Bioinformatics 30, 1312-1313 (2014).

21. H. J. Bandelt, P. Forster, A. Röhl, Median-joining networks for inferring intraspecific phylogenies. Mol. Biol. Evol.

https:/doi.org/10.1093/oxfordjournals.molbev.a026036. 
22. G. Vaidya, D. J. Lohman, R. Meier, SequenceMatrix: Concatenation software for the fast assembly of multi-gene datasets with character set and codon information. Cladistics 27, 171-180 (2011).

23. A. J. Drummond, M. A. Suchard, D. Xie, A. Rambaut, Bayesian phylogenetics with BEAUti and the BEAST 1.7. Mol. Biol. Evol. 29, 1969-1973 (2012).

24. M. A. Miller, W. Pfeiffer, T. Schwartz, The CIPRES science gateway in Proceedings of the 1st Conference of the Extreme Science and Engineering Discovery Environment on Bridging from the EXtreme to the Campus and beyond - XSEDE '12, (ACM Press, 2012), p. 1.

25. E. Vynnycky, R. G. White, An introduction to infectious disease modelling (Oxford: Oxford University Press, 2010).

26. R. M. Anderson, R. M. May, Infectious diseases of humans: Dynamics and control (OUP Oxford, 1992).

27. A. W. Byrne, et al., Inferred duration of infectious period of SARS-CoV-2: rapid scoping review and analysis of available evidence for asymptomatic and symptomatic COVID19 cases. MedRxiv (2020) https:/doi.org/10.1101/2020.04.25.20079889.

28. B. Murrell, et al., FUBAR: a fast, unconstrained bayesian appRoximation for inferring selection. Mol. Biol. Evol. 30, 1196-1205 (2013).

29. B. Murrell, et al., Detecting individual sites subject to episodic diversifying selection. PLoS Genet. 8 (2012).

30. S. L. Kosakovsky Pond, S. D. W. Frost, S. V. Muse, HyPhy: Hypothesis testing using phylogenies. Bioinformatics 21, 676-679 (2005).

31. S. Woolley, J. Johnson, M. J. Smith, K. A. Crandall, D. A. McClellan, TreeSAAP: Selection on amino acid properties using phylogenetic trees. Bioinformatics 19, 671672 (2003).

32. D. A. McClellan, K. G. McCracken, Estimating the influence of selection on the variable amino acid sites of the cytochrome b protein functional domains . Mol. Biol. Evol. 18, 917-925 (2001).

33. D. L. Swofford, PAUP. Phylogenetic analysis using parsimony (and other methods). Version 4. Sinauer Assoc. Sunderland, Massachusetts., 294-307 (2002).

34. E. Minskaia, et al., Discovery of an RNA virus $3^{\prime} \rightarrow 5^{\prime}$ exoribonuclease that is critically involved in coronavirus RNA synthesis. Proc. Natl. Acad. Sci. 103, 5108-5113 (2006).

35. T. Gojobori, E. N. Moriyama, M. Kimura, Molecular clock of viral evolution, and the neutral theory. Proc. Natl. Acad. Sci. 87, 10015-10018 (1990).

36. P. Forster, L. Forster, C. Renfrew, M. Forster, Phylogenetic network analysis of SARSCoV-2 genomes. Proc. Natl. Acad. Sci. 117, 9241-9243 (2020).

37. Y. Bai, et al., Evolution and molecular characteristics of SARS-CoV-2 genome. bioRxiv 
(2020) https:/doi.org/10.1101/2020.04.24.058933.

38. Y.-R. Guo, et al., The origin, transmission and clinical therapies on coronavirus disease 2019 (COVID-19) outbreak - an update on the status. Mil. Med. Res. 7, 11 (2020).

39. F. Wu, et al., A new coronavirus associated with human respiratory disease in China. Nature 579, 265-269 (2020).

40. M. A. Shereen, S. Khan, A. Kazmi, N. Bashir, R. Siddique, COVID-19 infection: Origin, transmission, and characteristics of human coronaviruses. J. Adv. Res. 24, 91-98 (2020).

41. T.-M. Chen, et al., A mathematical model for simulating the phase-based transmissibility of a novel coronavirus. Infect. Dis. Poverty 9, 24 (2020).

42. K. Xiao, et al., Isolation of SARS-CoV-2-related coronavirus from Malayan pangolins. Nature (2020) https:/doi.org/10.1038/s41586-020-2313-x.

43. R. Sanjuán, M. R. Nebot, N. Chirico, L. M. Mansky, R. Belshaw, Viral mutation rates. J. Virol. 84, 9733-9748 (2010).

44. Z. Zhao, et al., Moderate mutation rate in the SARS coronavirus genome and its implications. BMC Evol. Biol. 4, 21 (2004).

45. M. Vignuzzi, J. K. Stone, J. J. Arnold, C. E. Cameron, R. Andino, Quasispecies diversity determines pathogenesis through cooperative interactions in a viral population. Nature 439, 344-348 (2006).

46. C. Huang, et al., Clinical features of patients infected with 2019 novel coronavirus in Wuhan, China. Lancet 395, 497-506 (2020).

47. L. Ferretti, et al., Quantifying SARS-CoV-2 transmission suggests epidemic control with digital contact tracing. Science 368, eabb6936 (2020).

48. T. K. Tsang, et al., Effect of changing case definitions for COVID-19 on the epidemic curve and transmission parameters in mainland China: a modelling study. Lancet Public Heal. (2020) https:/doi.org/10.1016/S2468-2667(20)30089-X.

49. K. Mizumoto, K. Kagaya, G. Chowell, Early epidemiological assessment of the transmission potential and virulence of coronavirus disease 2019 (COVID-19) in Wuhan City: China, January-February, 2020. MedRxiv (2020) https:/doi.org/10.1101/2020.02.12.20022434

50. S. Sanche, et al., High contagiousness and rapid spread of severe acute respiratory syndrome coronavirus 2. Emerg. Infect. Dis. 26 (2020).

51. P. Serrano, et al., Nuclear magnetic resonance structure of the nucleic acid-binding domain of severe acute respiratory syndrome coronavirus nonstructural protein 3. $\mathrm{J}$. Virol. 83, 12998-13008 (2009).

52. S. Baliji, S. A. Cammer, B. Sobral, S. C. Baker, Detection of nonstructural protein 6 in murine coronavirus-infected cells and analysis of the transmembrane topology by using 
bioinformatics and molecular approaches. J. Virol. 83, 6957-6962 (2009).

53. D. Benvenuto, et al., Evolutionary analysis of SARS-CoV-2: how mutation of nonstructural protein 6 (NSP6) could affect viral autophagy. J. Infect. (2020) https:/doi.org/10.1016/j.jinf.2020.03.058.

54. M. R. Valentine, J. Termini, Kinetics of formation of hypoxanthine containing base pairs by HIV-RT: RNA template effects on the base substitution frequencies. Nucleic Acids Res. 29, 1191-1199 (2001). 


\section{Figure and Table Legends}

Figure 1. Phylogenetic tree of SARS-CoV-2 reconstructed from the concatenated dataset of all peptide encoding sequences using Bayesian Inference. Support values (posterior probabilities) of main clades/subclades are shown. Coloured branches refer to geographic locations: China (blue), America (red), Asia (orange), Europe (green), Australia (pink), Africa (cyan). The sample of bat-RaTG13-CoV was used as outgroup.

Figure 2. Median-joining network constructed using whole genome dataset of 25 SARS-CoV2 and bat-RaTG13-CoV samples. A detailed explanation on the information about samples was given in Table S2. The marked colours: cyan (Guangdong Province), lilac (Wuhan Province), blue (other Chinese samples), red (America), brown (Asia). The length of links was proportional to the number of mutated nucleotide positions between two circles and open circles indicate missing haplotypes.

Figure 3. Median estimation of tMRCA of SARS-CoV-2 inferred from Bayesian coalescent model using demographic reconstruction option. the dataset was derived from an aligment of all peptide encoding sequences of 821 SARS-CoV-2 samples. An exponentially growing population rate mode of demography and a GTR + G model of substitution were assumed. The $x$ axis is given as units of years, and the $y$ axis is equal to the product of the effective population size. The bold dashed line is the median estimate, and the thick dashed lines display the 95\% HPD limits (see text for details).

Figure 4. The codon patterns in terms of the magnitude of $d N-d S$ (nonsynonymous synonymous substitutions per site) of all peptide encoding sequences of SARS-CoV-2 samples (821 samples) in FUBAR analysis. Neutral and/or nearly neutral evolving codons were shown with red colour (that over there the magnitude of $d \mathrm{~N}-\mathrm{dS}$ is between -1 to +1 ). The codons subject to positive selection ( $d N-d S>1)$ were shown with white and to purifying selection $(\mathrm{dN}-\mathrm{dS}<1)$ were shown with black.

Figure 5. The substitution types and distribution patterns of synapomorphic changes defining the clades (or branches) of human SARS-CoV-2 and bat-RaTG13-CoV. a) The distribution graph of transitions and transversions throughout the genomes, b) the number of transitiontype differences (GA, AG, CT, TC), c) the number of transversion-type differences (CA, AC, TG, GT, AT, TA, GC, CG) 
Table 1. Divergence values of each peptide encoding sequences (10 CDS and $16 \mathrm{nsps}$ synthesised from orf1ab) and overall genome of SARS-CoV-2.

Table 2. Nucleotide composition of each peptide encoding sequences (10 CDS and $16 \mathrm{nsps}$ synthesised from orf1ab) and overall genome of SARS-CoV-2.

Table 3. The substitution rate estimations of each peptide encoding sequences (10 CDS and 16 nsps synthesised from orf1ab) and overall genome based on the available dates for samples using an uncorrelated relaxed molecular clock model with an exponentially growing population in BEAST, mean values \pm standard deviations.

Table 4. The codons under pervasive and/or episodic positive selection of each peptide encoding sequences of SARS-CoV-2 and their magnitude categories (1-2: conservative, 3-5: moderate, and 6-8: radical) and physicochemical properties detected by TreeSAAP. 
Table 1.

\begin{tabular}{|c|c|c|c|c|c|c|c|c|}
\hline \multirow{2}{*}{ Gene } & \multirow{2}{*}{$\begin{array}{l}\text { Length } \\
\text { (nt) }\end{array}$} & \multicolumn{4}{|c|}{ Nucleotide divergence } & \multirow{2}{*}{$\begin{array}{c}\text { a.a } \\
\text { divergence }\end{array}$} & \multicolumn{2}{|c|}{$\begin{array}{l}\text { Nucleotide } \\
\text { diversity ( } \pi)\end{array}$} \\
\hline & & overall & $\begin{array}{c}\text { non- } \\
\text { degenera } \\
\text { te }\end{array}$ & $\begin{array}{c}\text { twofold } \\
\text { degenerate }\end{array}$ & $\begin{array}{c}\text { fourfold } \\
\text { degenera } \\
\text { te }\end{array}$ & & dist. & stdev \\
\hline All genes & 29238 & 0.029 & 0.025 & 0.034 & 0.028 & 0.057 & 0.00028 & 0.00004 \\
\hline Leader protein & 540 & 0.041 & 0.025 & 0.081 & 0.044 & 0.067 & 0.00036 & 0.00020 \\
\hline nsp2 & 1914 & 0.038 & 0.035 & 0.049 & 0.038 & 0.075 & 0.00034 & 0.00010 \\
\hline nsp3 & 5835 & 0.026 & 0.025 & 0.029 & 0.019 & 0.059 & 0.00019 & 0.00008 \\
\hline nsp4 & 1500 & 0.030 & 0.023 & 0.043 & 0.028 & 0.048 & 0.00037 & 0.00023 \\
\hline 3C-like proteinase & 918 & 0.024 & 0.018 & 0.040 & 0.029 & 0.036 & 0.00008 & 0.00002 \\
\hline nsp6 & 873 & 0.039 & 0.033 & 0.077 & 0.000 & 0.072 & 0.00036 & 0.00024 \\
\hline nsp7 & 249 & 0.032 & 0.025 & 0.059 & 0.029 & 0.048 & 0.00009 & 0.00003 \\
\hline nsp8 & 594 & 0.017 & 0.015 & 0.009 & 0.018 & 0.040 & 0.00007 & 0.00002 \\
\hline nsp9 & 339 & 0.018 & 0.014 & 0.029 & 0.019 & 0.027 & 0.00005 & 0.00002 \\
\hline nsp10 & 417 & 0.031 & 0.025 & 0.000 & 0.077 & 0.058 & 0.00009 & 0.00003 \\
\hline nsp11 & 39 & 0.051 & 0.000 & 0.143 & 0.143 & 0.077 & 0.00013 & 0.00009 \\
\hline RdRP & 2796 & 0.020 & 0.018 & 0.026 & 0.022 & 0.043 & 0.00027 & 0.00027 \\
\hline Helicase & 1803 & 0.022 & 0.022 & 0.017 & 0.033 & 0.042 & 0.00035 & 0.00015 \\
\hline $\begin{array}{l}3^{\prime}-\text {-to-5' } \\
\text { exonuclease }\end{array}$ & 1581 & 0.016 & 0.014 & 0.012 & 0.039 & 0.028 & 0.00019 & 0.00014 \\
\hline EndoRNase & 1038 & 0.021 & 0.021 & 0.027 & 0.015 & 0.046 & 0.00010 & 0.00003 \\
\hline $\begin{array}{l}\text { 2'-O-ribose } \\
\text { methyltransferase }\end{array}$ & 897 & 0.021 & 0.019 & 0.027 & 0.025 & 0.033 & 0.00006 & 0.00001 \\
\hline S gene & 3819 & 0.030 & 0.028 & 0.030 & 0.028 & 0.061 & 0.00025 & 0.00010 \\
\hline ORF3a & 825 & 0.047 & 0.041 & 0.058 & 0.035 & 0.105 & 0.00045 & 0.00022 \\
\hline E gene & 225 & 0.070 & 0.050 & 0.137 & 0.051 & 0.120 & 0.00023 & 0.00006 \\
\hline M gene & 666 & 0.029 & 0.019 & 0.039 & 0.058 & 0.041 & 0.00039 & 0.00022 \\
\hline ORF6 & 183 & 0.016 & 0.000 & 0.065 & 0.000 & 0.033 & 0.00020 & 0.00006 \\
\hline ORF7a & 363 & 0.014 & 0.009 & 0.040 & 0.000 & 0.025 & 0.00003 & 0.00001 \\
\hline ORF7b & 129 & 0.016 & 0.024 & 0.000 & 0.000 & 0.047 & 0.00004 & 0.00003 \\
\hline ORF8 & 363 & 0.033 & 0.030 & 0.049 & 0.000 & 0.083 & 0.00115 & 0.00103 \\
\hline $\mathrm{N}$ gene & 1257 & 0.058 & 0.046 & 0.074 & 0.058 & 0.110 & 0.00094 & 0.00031 \\
\hline ORF10 & 114 & 0.070 & 0.040 & 0.044 & 0.214 & 0.079 & 0.00028 & 0.00009 \\
\hline
\end{tabular}


Table 2.

\begin{tabular}{|c|c|c|c|c|c|c|c|c|c|c|c|}
\hline \multirow{2}{*}{ Gene } & \multicolumn{5}{|c|}{ Nucleotide composition } & \multirow{2}{*}{ Gene } & \multicolumn{5}{|c|}{ Nucleotide composition } \\
\hline & & U\% & $\mathrm{C} \%$ & $\mathbf{A} \%$ & G\% & & & U\% & $\mathrm{C} \%$ & $A \%$ & G\% \\
\hline & Avg. & 32.22 & 18.34 & 29.82 & 19.62 & & Avg. & 32.39 & 18.27 & 29.35 & 19.99 \\
\hline All & 1. codon & 22.95 & 16.49 & 30.10 & 30.46 & 3'-to-5' & 1. codon & 23.15 & 17.46 & 29.22 & 30.17 \\
\hline \multirow[t]{3}{*}{ genes } & 2.codon & 30.09 & 22.91 & 31.29 & 15.71 & \multirow[t]{3}{*}{ exonuclease } & 2.codon & 28.85 & 20.87 & 32.07 & 18.22 \\
\hline & 3. codon & 43.63 & 15.61 & 28.08 & 12.68 & & 3. codon & 45.18 & 16.49 & 26.76 & 11.57 \\
\hline & Avg. & 26.47 & 21.50 & 25.00 & 27.04 & & Avg. & 32.85 & 14.45 & 33.14 & 19.56 \\
\hline Leader & 1. codon & 12.22 & 26.11 & 18.89 & 42.78 & \multirow{3}{*}{ EndoRNase } & 1. codon & 20.52 & 14.16 & 30.06 & 35.26 \\
\hline \multirow[t]{3}{*}{ protein } & 2.codon & 29.44 & 14.45 & 33.89 & 22.22 & & 2.codon & 33.53 & 18.21 & 34.68 & 13.58 \\
\hline & 3. codon & 37.74 & 23.93 & 22.23 & 16.11 & & 3. codon & 44.51 & 10.98 & 34.68 & 9.83 \\
\hline & Avg. & 30.31 & 18.44 & 29.83 & 21.42 & \multirow{3}{*}{$\begin{array}{l}\text { 2'-O-ribose } \\
\text { methyltransfer }\end{array}$} & Avg. & 33.00 & 16.00 & 31.54 & 19.46 \\
\hline \multirow{3}{*}{ nsp2 } & 1. codon & 21.32 & 15.20 & 30.10 & 33.37 & & 1. codon & 22.15 & 15.44 & 33.89 & 28.52 \\
\hline & 2.codon & 29.16 & 22.10 & 31.66 & 17.08 & & 2.codon & 30.87 & 21.81 & 31.54 & 15.77 \\
\hline & 3. codon & 40.44 & 18.02 & 27.75 & 13.79 & & 3. codon & 45.97 & 10.74 & 29.19 & 14.09 \\
\hline \multirow{4}{*}{ nsp3 } & Avg. & 31.99 & 16.75 & 32.12 & 19.14 & \multirow{4}{*}{ S gene } & Avg. & 33.26 & 18.93 & 29.40 & 18.42 \\
\hline & 1. codon & 21.85 & 14.60 & 32.13 & 31.41 & & 1. codon & 24.20 & 16.42 & 30.48 & 28.91 \\
\hline & 2.codon & 28.64 & 23.60 & 34.60 & 13.16 & & 2.codon & 29.23 & 24.43 & 30.77 & 15.58 \\
\hline & 3. codon & 45.47 & 12.07 & 29.61 & 12.85 & & 3. codon & 46.35 & 15.94 & 26.94 & 10.76 \\
\hline \multirow{4}{*}{ nsp4 } & Avg. & 36.95 & 17.72 & 26.60 & 18.73 & \multirow{4}{*}{ ORF3a } & Avg. & 33.35 & 21.09 & 27.03 & 18.53 \\
\hline & 1. codon & 28.40 & 13.60 & 28.80 & 29.20 & & 1. codon & 25.82 & 17.82 & 28.73 & 27.64 \\
\hline & 2.codon & 34.20 & 24.40 & 25.00 & 16.40 & & 2.codon & 34.22 & 23.27 & 28.00 & 14.51 \\
\hline & 3. codon & 48.25 & 15.15 & 26.00 & 10.60 & & 3. codon & 40.02 & 22.18 & 24.36 & 13.44 \\
\hline \multirow{5}{*}{$\begin{array}{c}\text { 3C-like } \\
\text { proteina } \\
\text { se }\end{array}$} & Avg. & 33.77 & 17.75 & 28.11 & 20.37 & \multirow{4}{*}{ E gene } & Avg. & 40.45 & 19 & 20.89 & 18.67 \\
\hline & 1. codon & 22.55 & 17.65 & 28.43 & 31.37 & & 1. codon & & & 24.00 & 28.00 \\
\hline & 2.codon & 30.72 & 21.89 & 29.41 & 17.97 & & 2.codon & & & 18.67 & 12.00 \\
\hline & 3. codon & 48.04 & 13.72 & 26.47 & 11.76 & & 3. codon & 45.34 & & 20.00 & 16.00 \\
\hline & Avg. & 39.56 & 16.55 & 24.14 & 19.76 & & Avg. & 31.84 & 21.91 & 25.37 & 20.87 \\
\hline & 1. codon & 30.69 & 13.45 & 30.34 & 25.52 & & 1. codon & 21.62 & 22.07 & 30.18 & 26.12 \\
\hline nsp6 & 2.codon & 42.76 & 20.69 & 21.03 & 15.52 & M gene & 2.codon & 36.97 & 20.68 & 22.07 & 20.27 \\
\hline & 3. codon & 45.22 & 15.51 & 21.03 & 18.23 & & 3. codon & 36.94 & 22.97 & 23.87 & 16.22 \\
\hline & Avg. & 30.12 & 19.68 & 31.73 & 18.47 & & Avg. & 35.52 & 14.21 & 36.07 & 14.21 \\
\hline & 1. codon & 26.51 & 18.07 & 25.30 & 30.12 & & 1. codon & 21.31 & 16.39 & 40.98 & 21.31 \\
\hline & 2.codon & 37.35 & 22.89 & 31.32 & 8.43 & & 2.codon & 44.26 & 14.75 & 37.70 & 3.28 \\
\hline & 3. codon & 26.51 & 18.07 & 38.56 & 16.87 & & 3. codon & 40.99 & 11.48 & 29.51 & 18.02 \\
\hline & Avg. & 29.13 & 18.01 & 32.49 & 20.37 & & Avg. & 32.23 & 21.76 & 29.48 & 16.53 \\
\hline & 1. codon & 17.17 & 14.14 & 35.35 & 33.33 & & 1. codon & 24.79 & 22.31 & 27.27 & 25.62 \\
\hline nsp8 & 2.codon & 27.78 & 28.78 & 33.84 & 9.60 & ORF7a & 2.codon & 34.71 & 25.62 & 26.45 & 13.22 \\
\hline & 3. codon & 42.43 & 11.11 & 28.28 & 18.18 & & 3. codon & 37.19 & 17.35 & 34.71 & 10.75 \\
\hline & Avg. & 28.91 & 18.88 & 30.97 & 21.24 & & Avg. & 45.74 & 18.60 & 22.48 & 13.18 \\
\hline & 1. codon & 19.47 & 17.70 & 32.74 & 30.09 & & 1. codon & 37.21 & 23.26 & 20.93 & 18.60 \\
\hline nsps & 2.codon & 25.66 & 26.55 & 28.32 & 19.47 & ORF $/ \mathrm{b}$ & 2.codon & 58.14 & 11.63 & 23.26 & 6.98 \\
\hline & 3. codon & 41.59 & 12.39 & 31.86 & 14.16 & & 3. codon & 43.00 & 41.86 & 20.93 & 23.26 \\
\hline & Avg. & 29.74 & 20.14 & 27.82 & 22.30 & & Avg. & 36.57 & 17.70 & 27.27 & 18.45 \\
\hline & 1. codon & 22.30 & 17.27 & 27.34 & 33.09 & & 1. codon & 31.40 & 17.37 & 22.31 & 28.92 \\
\hline nsp10 & 2.codon & 21.59 & 27.33 & 28.78 & 22.30 & ORF- & 2.codon & 33.68 & 20.04 & 30.58 & 15.70 \\
\hline & 3. codon & 45.32 & 15.83 & 27.34 & 11.51 & & 3. codon & 44.63 & 15.70 & 28.93 & 10.75 \\
\hline & Avg. & 33.33 & 17.95 & 20.51 & 28.21 & & Avg. & 21.01 & 25.07 & 31.69 & 22.24 \\
\hline & 1. codon & 38.46 & 7.69 & 7.69 & 46.16 & & 1. codon & 14.79 & 24.15 & 31.50 & 29.56 \\
\hline nsp11 & 2.codon & 30.76 & 38.47 & 23.08 & 7.69 & N gene & 2.codon & 16.48 & 28.39 & 33.21 & 21.92 \\
\hline & 3. codon & 30.78 & 7.70 & 30.75 & 30.77 & & 3. codon & 31.75 & 22.66 & 30.35 & 15.24 \\
\hline & Avg. & 32.45 & 17.94 & 30.26 & 19.35 & & Avg. & 35.97 & 18.42 & 29.81 & 15.80 \\
\hline & 1. codon & 24.46 & 15.77 & 28.76 & 31.01 & & 1. codon & 26.31 & 15.80 & 36.85 & 21.05 \\
\hline RdRP & 2.codon & 29.22 & 20.35 & 34.98 & 15.45 & ORF10 & 2.codon & 44.74 & 15.81 & 26.27 & 13.18 \\
\hline & 3. codon & 43.68 & 17.70 & 27.04 & 11.59 & & 3. codon & 36.86 & 23.66 & 26.32 & 13.16 \\
\hline
\end{tabular}

$\begin{array}{llllll}\text { Helicase } & 1 . \text { codon } & 23.30 & 16.63 & 29.62 & 30.45 \\ & \text { 2.codon } & 27.31 & 25.77 & 29.93 & 16.99\end{array}$


Table 3.

\begin{tabular}{|c|c|c|c|c|c|}
\hline Gene & Mean Rate & ucld.mean & ucld.stdev & $\begin{array}{c}\text { Coefficient } \\
\text { Variance }\end{array}$ & Covariance \\
\hline Overall & $1.6463 \mathrm{E}-3 \pm 1.1729 \mathrm{E}-3$ & $1.8553 \mathrm{E}-3 \pm 1.8089 \mathrm{E}-4$ & $3.1755 \mathrm{E}-3 \pm 6.4253 \mathrm{E}-4$ & $1.5201 \pm 0.1616$ & $9.6540 \mathrm{E}-3 \pm 0.0263$ \\
\hline leader protein & $1.1490 \mathrm{E}-2 \pm 3.5338 \mathrm{E}-3$ & $1.4910 \mathrm{E}-2 \pm 5.1791 \mathrm{E}-3$ & $3.2020 \mathrm{E}-1 \pm 0.2843$ & $6.0377 \pm 1.9809$ & $-1.041 \mathrm{E}-3 \pm 0.0225$ \\
\hline nsp2 & $3.4289 \mathrm{E}-3 \pm 7.4638 \mathrm{E}-4$ & $3.5720 \mathrm{E}-3 \pm 7.9356 \mathrm{E}-4$ & $5.2479 \mathrm{E}-3 \pm 3.345 \mathrm{E}-3$ & $1.3393 \pm 0.5961$ & $-6.353 E-4 \pm 0.0243$ \\
\hline nsp3 & $2.5571 \mathrm{E}-3 \pm 4.2484 \mathrm{E}-4$ & $2.9112 \mathrm{E}-3 \pm 6.4867 \mathrm{E}-4$ & $1.3100 \mathrm{E}-2 \pm 8.958 \mathrm{E}-3$ & $3.1842 \pm 0.7727$ & $1.8389 \mathrm{E}-3 \pm 0.0263$ \\
\hline nsp4 & $3.8377 \mathrm{E}-3 \pm 8.6322 \mathrm{E}-4$ & $4.9686 \mathrm{E}-3 \pm 1.7065 \mathrm{E}-3$ & $7.3340 \mathrm{E}-2 \pm 0.1091$ & $4.2276 \pm 1.6733$ & $8.5638 \mathrm{E}-3 \pm 0.0295$ \\
\hline 3C-like proteinase & $4.0878 \mathrm{E}-4 \pm 3.8422 \mathrm{E}-4$ & $1.0579 \mathrm{E}-3 \pm 9.5410 \mathrm{E}-4$ & $2.4720 \mathrm{E}-1 \pm 0.2932$ & $10.9518 \pm 2.7896$ & $-1.965 E-3 \pm 0.0225$ \\
\hline nsp6 & $2.6641 \mathrm{E}-3 \pm 8.0519 \mathrm{E}-4$ & $4.3889 \mathrm{E}-3 \pm 1.7231 \mathrm{E}-3$ & $3.0710 \mathrm{E}-1 \pm 0.2907$ & $8.4642 \pm 2.6019$ & $-1.572 \mathrm{E}-3 \pm 0.0209$ \\
\hline nsp7 & $2.9584 \mathrm{E}-3 \pm 2.5303 \mathrm{E}-3$ & $3.1058 \mathrm{E}-3 \pm 2.3132 \mathrm{E}-3$ & $4.7950 \mathrm{E}-1 \pm 0.3769$ & $13.5180 \pm 2.6972$ & $-5.129 \mathrm{E}-4 \pm 0.0216$ \\
\hline nsp8 & $7.6830 \mathrm{E}-4 \pm 4.0444 \mathrm{E}-4$ & $1.5420 \mathrm{E}-3 \pm 9.1436 \mathrm{E}-4$ & $2.1360 \mathrm{E}-1 \pm 0.2731$ & $8.0462 \pm 3.5509$ & $-5.88 \mathrm{E}-4 \pm 0.0222$ \\
\hline nsp9 & $5.4660 \mathrm{E}-4 \pm 2.7272 \mathrm{E}-4$ & $1.1240 \mathrm{E}-3 \pm 6.3374 \mathrm{E}-4$ & $2.4500 \mathrm{E}-1 \pm 0.2879$ & $9.8006 \pm 4.1811$ & $-5.265 E-4 \pm 0.0212$ \\
\hline nsp10 & $4.6859 \mathrm{E}-3 \pm 3.4210 \mathrm{E}-3$ & $6.3142 \mathrm{E}-3 \pm 4.3439 \mathrm{E}-3$ & $2.5760 \mathrm{E}-1 \pm 0.2771$ & $7.0938 \pm 2.7221$ & $-7.177 \mathrm{E}-4 \pm 0.0219$ \\
\hline nsp11 & $2.5711 \mathrm{E}-4 \pm 2.3253 \mathrm{E}-4$ & $5.0020 \mathrm{E}-4 \pm 4.4155 \mathrm{E}-4$ & $4.0270 \mathrm{E}-1 \pm 0.3603$ & $15.7908 \pm 4.1241$ & $-7.069 \mathrm{E}-4 \pm 0.0201$ \\
\hline RdRP & $2.1835 \mathrm{E}-3 \pm 4.2071 \mathrm{E}-4$ & $2.7701 \mathrm{E}-3 \pm 8.5924 \mathrm{E}-4$ & $2.7690 \mathrm{E}-2 \pm 0.0464$ & $3.7833 \pm 1.3437$ & $-1.678 \mathrm{E}-3 \pm 0.0224$ \\
\hline Helicase & $1.6190 \mathrm{E}-3 \pm 4.6816 \mathrm{E}-4$ & $1.8098 \mathrm{E}-3 \pm 6.0089 \mathrm{E}-4$ & $7.4440 \mathrm{E}-3 \pm 0.0264$ & $2.0107 \pm 1.3527$ & $8.1003 \mathrm{E}-4 \pm 0.025$ \\
\hline 3'-to-5' exonuclease & $1.7954 \mathrm{E}-3 \pm 6.9984 \mathrm{E}-4$ & $2.2426 \mathrm{E}-3 \pm 1.0392 \mathrm{E}-3$ & $2.6220 \mathrm{E}-2 \pm 0.0723$ & $2.8530 \pm 1.0385$ & $2.1185 \mathrm{E}-3 \pm 0.025$ \\
\hline EndoRNase & $1.2974 \mathrm{E}-3 \pm 6.3716 \mathrm{E}-4$ & $1.5266 \mathrm{E}-3 \pm 8.8114 \mathrm{E}-4$ & $1.9800 \mathrm{E}-2 \pm 0.0712$ & $2.3566 \pm 2.0786$ & $2.727 \mathrm{E}-5 \pm 0.0287$ \\
\hline 2'-O-ribose methyltransferase & $1.3558 \mathrm{E}-3 \pm 8.0732 \mathrm{E}-4$ & $2.3030 \mathrm{E}-3 \pm 1.3548 \mathrm{E}-3$ & $1.8470 \mathrm{E}-1 \pm 0.2444$ & $7.2491 \pm 3.2383$ & $3.0817 \pm 0.0268$ \\
\hline S-gene & $3.7676 \mathrm{E}-3 \pm 6.1808 \mathrm{E}-4$ & $4.0959 \mathrm{E}-3 \pm 7.7225 \mathrm{E}-4$ & $1.0240 \mathrm{E}-2 \pm 5.211 \mathrm{E}-3$ & $2.2647 \pm 0.6117$ & $1.2223 \pm 0.0323$ \\
\hline orf3a & $2.8431 \mathrm{E}-3 \pm 9.1291 \mathrm{E}-4$ & $3.4825 \mathrm{E}-3 \pm 1.4402 \mathrm{E}-3$ & $2.8590 \mathrm{E}-2 \pm 0.0591$ & $3.0399 \pm 1.6010$ & $3.753 \mathrm{E}-4 \pm 0.0243$ \\
\hline E-gene & $8.4246 \mathrm{E}-3 \pm 4.6670 \mathrm{E}-4$ & $9.7163 \mathrm{E}-2 \pm 5.3637 \mathrm{E}-4$ & $3.6250 \mathrm{E}-1 \pm 0.3124$ & $8.7000 \pm 2.6760$ & $3.203 E-3 \pm 0.0279$ \\
\hline M-gene & $3.6035 \mathrm{E}-3 \pm 1.4276 \mathrm{E}-3$ & $5.3685 \mathrm{E}-3 \pm 2.3180 \mathrm{E}-3$ & $1.6700 \mathrm{E}-1 \pm 0.2139$ & $5.7807 \pm 2.4889$ & $-1.257 \mathrm{E}-3 \pm 0.0223$ \\
\hline orf6 & $1.3291 \mathrm{E}-3 \pm 9.1878 \mathrm{E}-4$ & $2.0736 \mathrm{E}-3 \pm 1.4197 \mathrm{E}-4$ & $2.8970 \mathrm{E}-1 \pm 0.3148$ & $9.8280 \pm 3.9884$ & $-9.472 E-4 \pm 0.0192$ \\
\hline orf7a & $2.9930 \mathrm{E}-4 \pm 3.1611 \mathrm{E}-4$ & $6.7744 \mathrm{E}-4 \pm 6.5021 \mathrm{E}-4$ & $3.1320 \mathrm{E}-1 \pm 0.3243$ & $12.0949 \pm 4.2280$ & $4.066 \mathrm{E}-4 \pm 0.0233$ \\
\hline orf7b & $9.0770 \mathrm{E}-4 \pm 7.8061 \mathrm{E}-4$ & $1.5165 \mathrm{E}-3 \pm 1.1948 \mathrm{E}-3$ & $3.4120 \mathrm{E}-1 \pm 0.3362$ & $11.7739 \pm 3.9525$ & $-2.678 \mathrm{E}-4 \pm 0.0233$ \\
\hline orf8 & $8.2470 \mathrm{E}-3 \pm 2.8474 \mathrm{E}-4$ & $1.0830 \mathrm{E}-2 \pm 4.3398 \mathrm{E}-3$ & $2.5680 \mathrm{E}-1 \pm 0.2676$ & $5.9695 \pm 2.3771$ & $-6.743 E-4 \pm 0.0233$ \\
\hline $\mathrm{N}$-gene & $4.5613 \mathrm{E}-3 \pm 1.4342 \mathrm{E}-3$ & $5.0338 \mathrm{E}-3 \pm 1.6280 \mathrm{E}-3$ & $2.0380 \mathrm{E}-2 \pm 0.0169$ & $2.9106 \pm 0.8869$ & $-2.968 \mathrm{E}-3 \pm 0.0232$ \\
\hline orf10 & $7.4580 \mathrm{E}-3 \pm 3.4741 \mathrm{E}-3$ & $9.4569 \mathrm{E}-3 \pm 4.4590 \mathrm{E}-3$ & $2.5140 \mathrm{E}-1 \pm 0.2815$ & $5.9868 \pm 2.9352$ & $-6.102 E-4 \pm 0.0226$ \\
\hline
\end{tabular}




\section{Supplementary Files}

\section{Supplementary Figures}

Figure S1. The phylogenetic tree reconstructed for defining synapomorphies at the clades (or branches) leading to human SARS-CoV-2 and bat-RaTG13-CoV. The sampling list used in the reconstruction of the tree was presented in Table S3.

\section{Supplementary Tables}

Table S1. Detailed description of the sampling list retrieved from GISAID database.

Table S2. A summary of the sampling list used in the network reconstruction of SARS-CoV-2

Tables3. A summary of the sampling list used in the phylogenetic tree reconstructed for defining synapomorphies at the clades (or branches) leading to human SARS-CoV-2 and batRaTG13-CoV

TableS4. The list of molecular synapomorphic characters that define the clades (or branches) of human SARS-CoV-2 and bat-RaTG13-CoV 


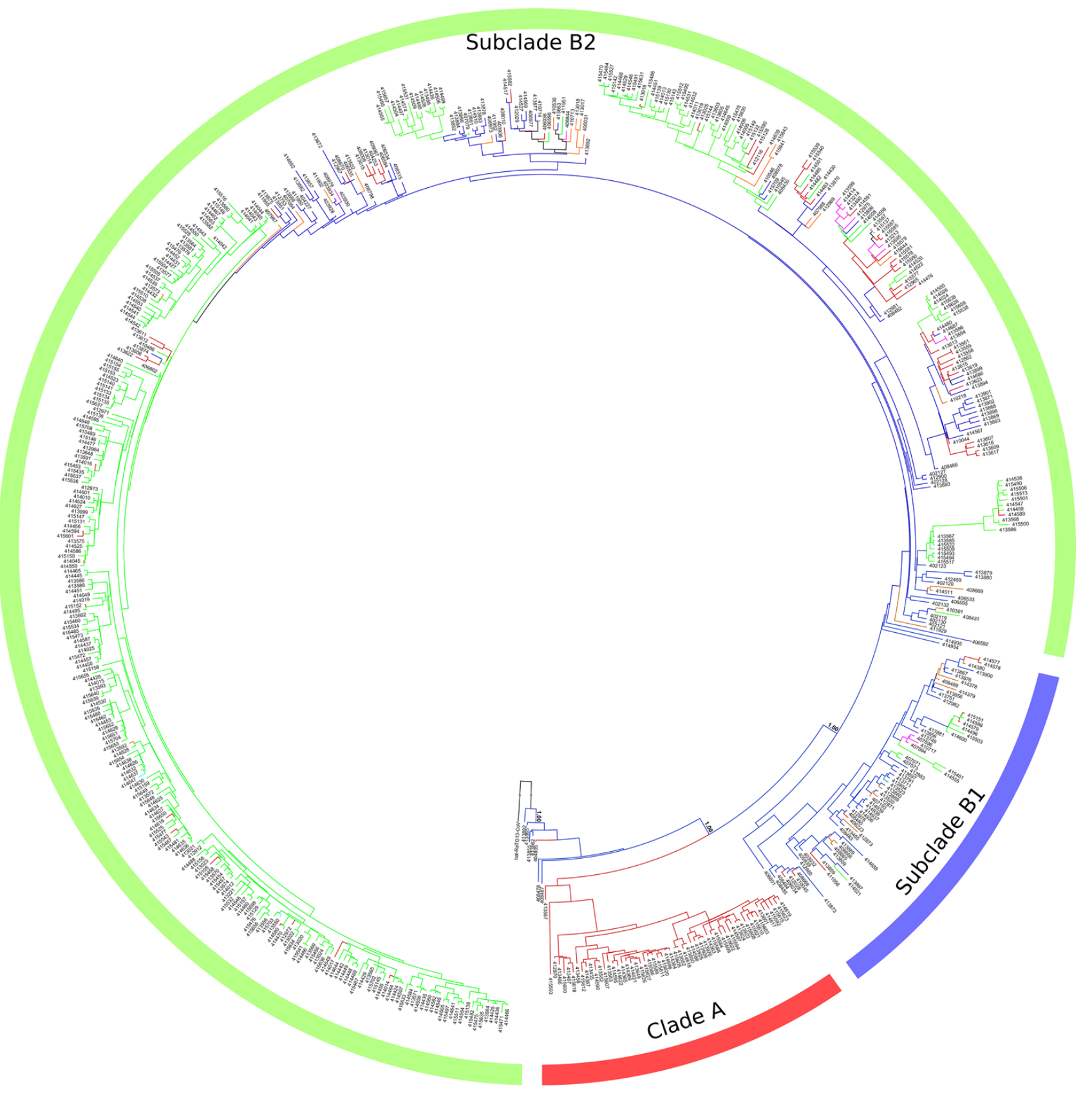

Figure 1 


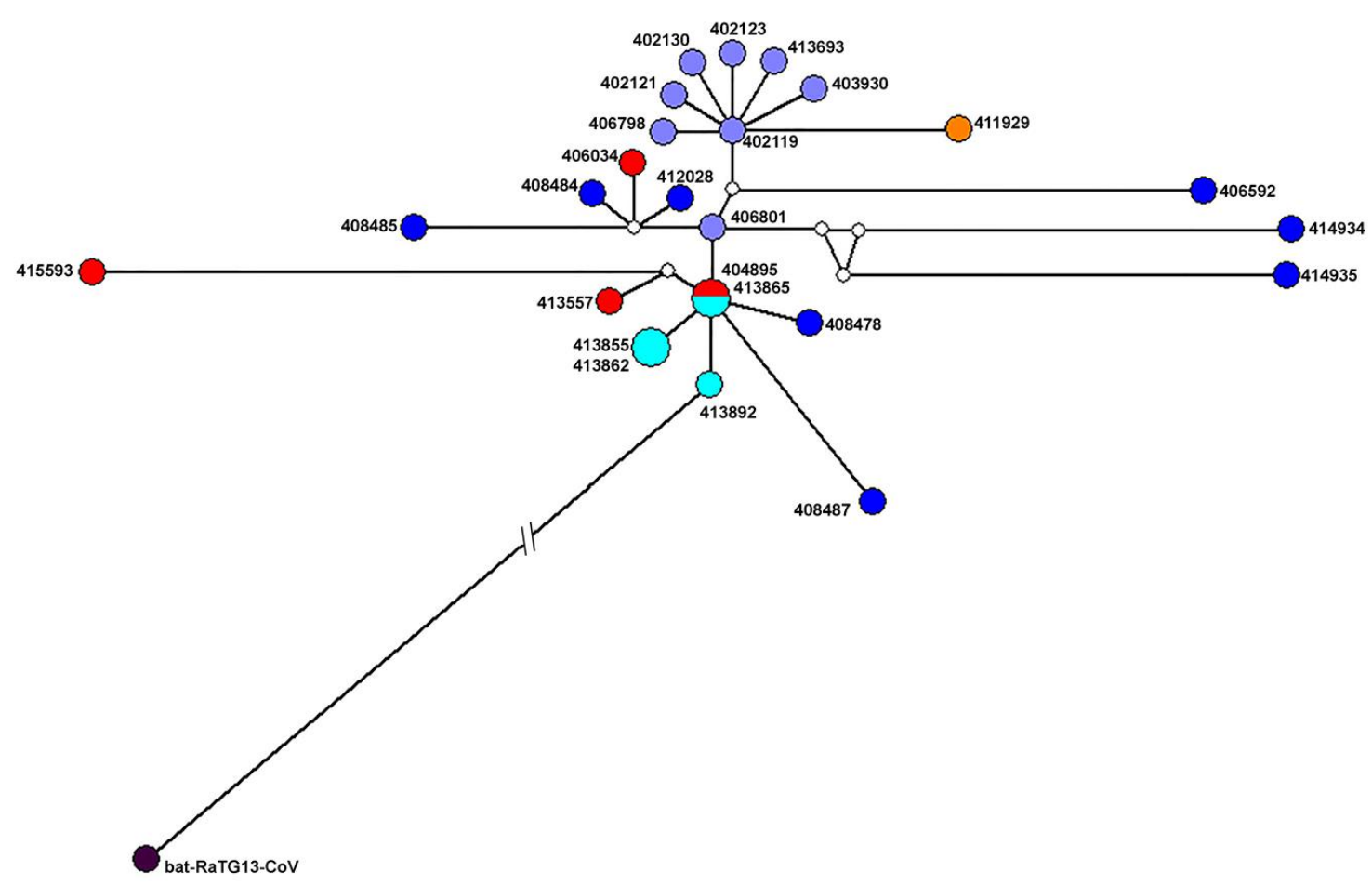

Figure 2 


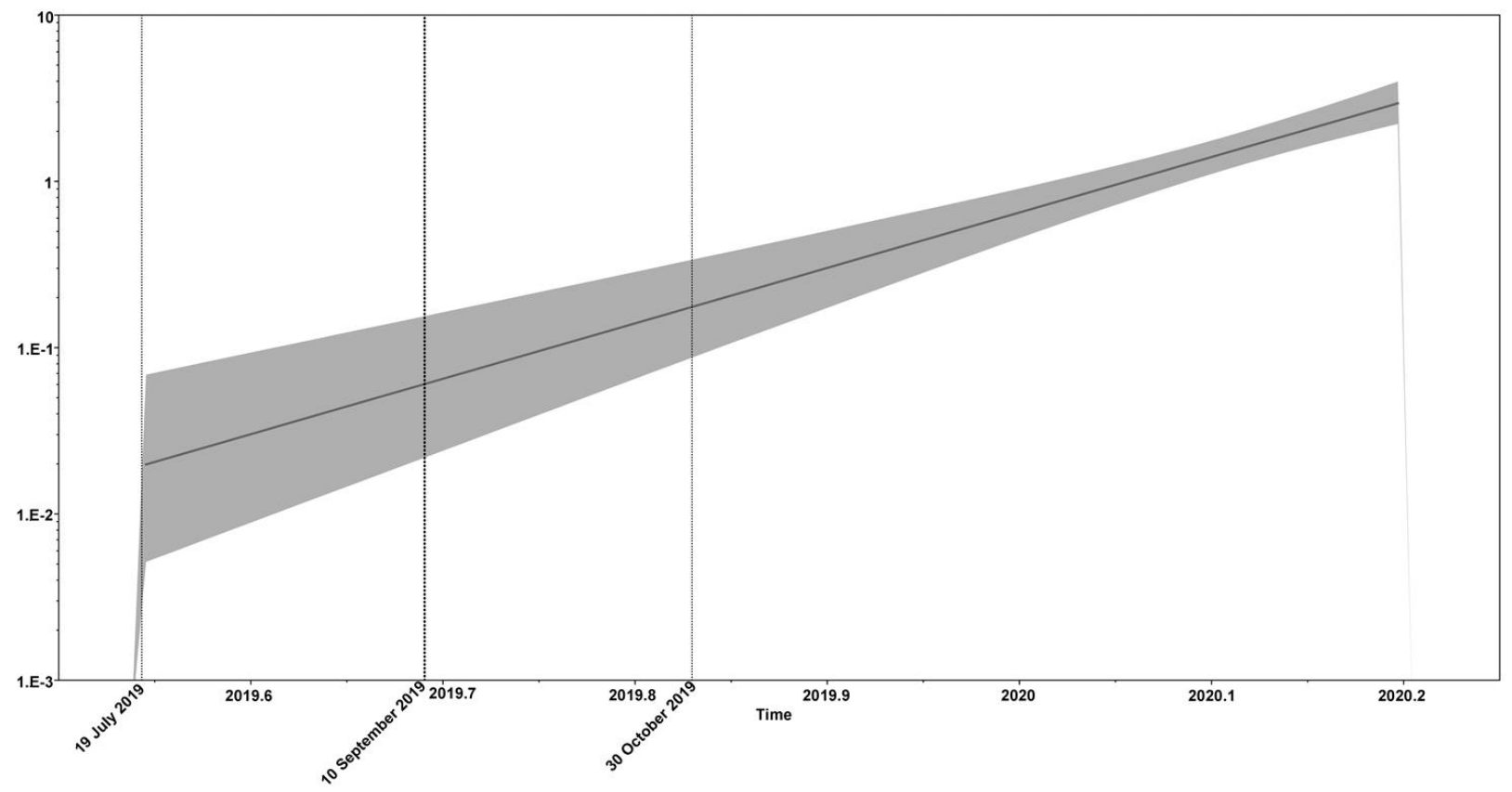

Figure 3 


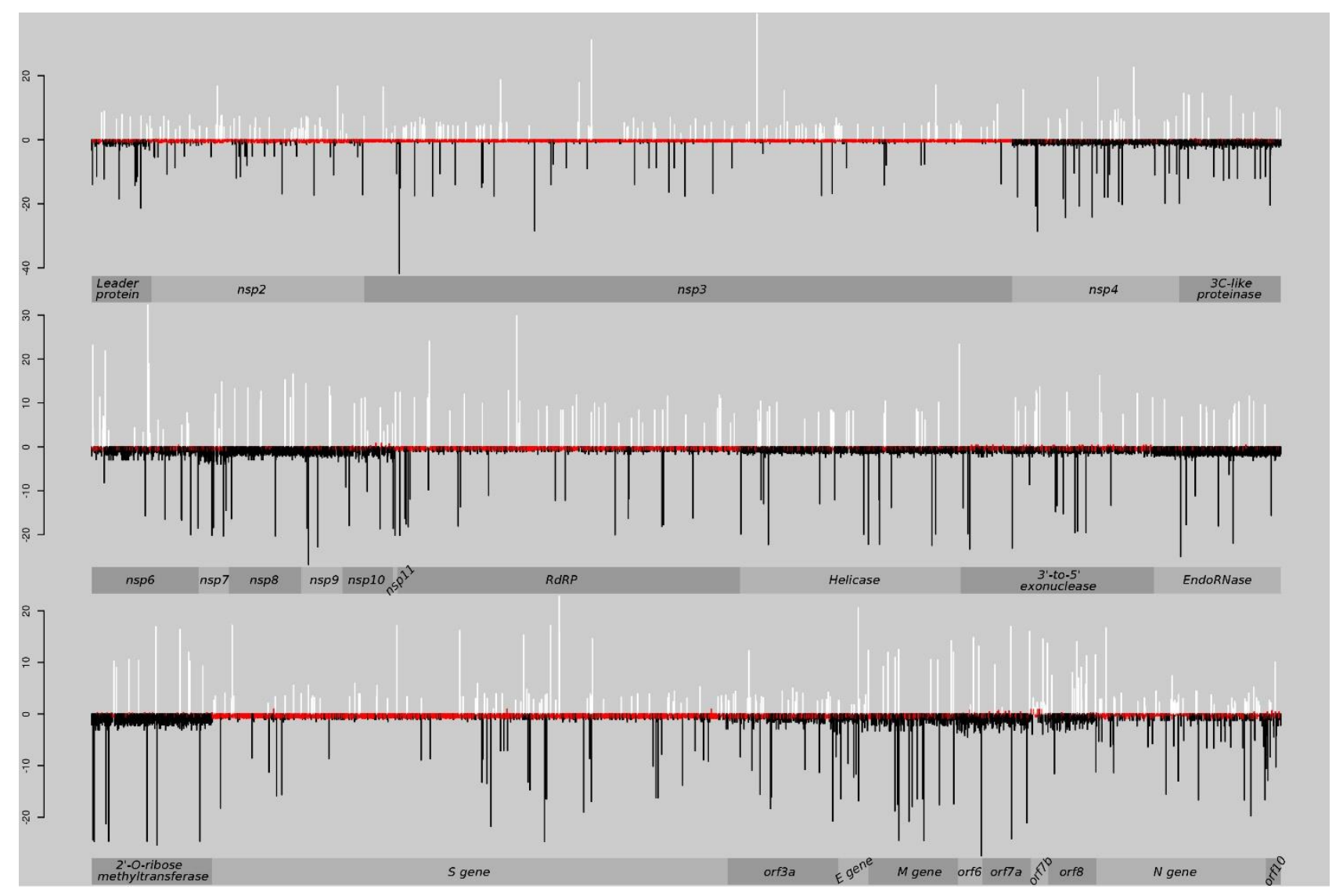

Figure 4 

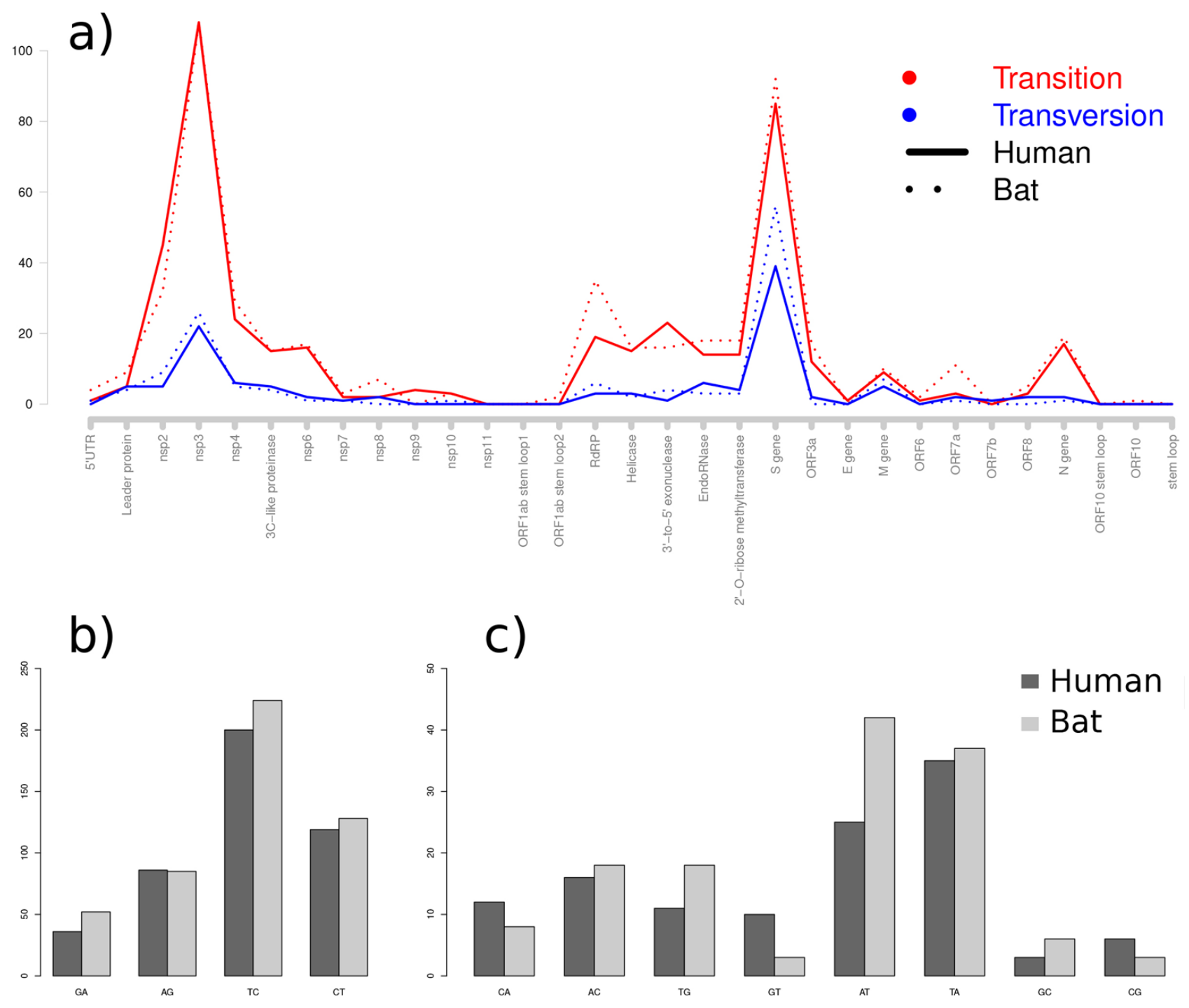

Figure 5 